\title{
ERRATUM
}

\section{Compile-Time Energy Optimization for Parallel Applications in On-Chip Multiprocessors}

\author{
Juan Chen ${ }^{1}$, Huizhan $\mathrm{Yi}^{1}$, Xuejun $\mathrm{Yang}^{1}$, and Liang Qian ${ }^{2}$ \\ ${ }^{1}$ School of Computer, National University of Defense Technology, \\ Changsha 410073, P.R.China \\ \{juanchen, huizhanyi, xjyang\} @nudt.edu.cn \\ ${ }^{2}$ Interdisciplinary Research Centre in Materials, University of Birmingham, UK \\ liangqian99@gmail.com
}

V.N. Alexandrov et al. (Eds.): ICCS 2006, Part II, LNCS 3992, pp. 904-911, 2006.

(C) Springer-Verlag Berlin Heidelberg 2006

Erratum DOI: 10.1007/11758525_149

In the original version of this paper, equation in the 2nd line of page 908 was not correct.

where $E_{s y n}=10 \mu \mathrm{J}, t_{s y n}=50$ cycles. 